\title{
Chronic pancreatitis: the perspective of pain generation by neuroimmune interaction
}

\author{
P Di Sebastiano, F F di Mola, D E Bockman, H Friess, M W Büchler
}

Gut 2003;52:907-911

Chronic pancreatitis (CP) is an inflammatory, often painful, disease of the exocrine pancreas which leads to exocrine insufficiency. The pathophysiology of pain in $\mathrm{CP}$ is incompletely understood. Several hypotheses have been advanced, including pancreatic and extrapancreatic causes. Here, the different pain hypotheses are discussed and evidence is presented that neuroimmune interactions are significant in the pathogenesis of pain generation and inflammation in CP. A better understanding of the complex cellular and molecular mechanisms of neuroimmune interactions should offer possibilities for innovative therapy and long term disease prevention.

See end of article for authors' affiliations

\section{Correspondence to:} Dr M W Büchler, Department of General Surgery, University of Heidelberg, D-69120 Heidelberg, Germany; Markus_Buechler@ med.uni-heidelberg.de

Accepted for publication 4 February 2003

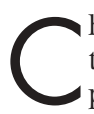

hronic pancreatitis (CP) is an inflammapancreas which leads to exocrine insufficiency. It affects approximately eight new patients per 100000 population per year in the USA, with a prevalence of 26.4 cases per 100000 population. ${ }^{1} \mathrm{CP}$ is characterised by parenchymal fibrosis, ductal strictures, and atrophy of acinar and islet tissue. ${ }^{2}$ The ongoing pancreatic destruction leads to varying degrees of maldigestion and in advanced stages to endocrine insufficiency. Complications encountered in CP include pancre(10-25\%) obstruction. However, the most clinically relevant feature of $\mathrm{CP}$ is recurrent upper abdominal pain. Pain can be so intense and long lasting that the follow up care of patients is difficult and frustrating. ${ }^{3}$ Attacks of acute pancreatitis may be superimposed on the pattern of chronic pain. Many patients become addicted to the narcotics that are prescribed. Several attempts have been made to evaluate the nature of pain in CP by using questionnaires and pain scales (for example, a visual analogue scale $)^{56}$ but it is difficult to obtain sufficient data as intensity, radiation, and duration are not constant.

The pathophysiology of pain in CP is incompletely understood. Several hypotheses have been advanced, including pancreatic and extrapancreatic causes. The existence of varied hypotheses to explain the genesis of pain in CP is reflected in the different approaches to treatment for pain relief in these patients. ${ }^{45}$ Increased intraductal pressure as a result of single or multiple strictures and/or calculi is believed to be a common cause of pain in CP patients with a dilated main pancreatic duct. Other suggested causes include pancreatic fibrosis, interstitial hypertension, and pancreatic tory, often painful, disease of the exocrine atic pseudocyst, and biliary (30\%) and duodenal ischaemia. ${ }^{78}$ Additionally, extrapancreatic causes such as duodenal and common bile duct stenosis with scarring due to pancreatic inflammation are suggested as factors causing pain in $\mathrm{CP}^{9-11} \mathrm{CP}$ may be asymptomatic from the first or, it has been hypothesised, pain may disappear when the disease burns itself out in late stages. ${ }^{12}$

In this review we will discuss different pain hypotheses and present the perspective that neuroimmune interactions are significant in pain generation in CP. In this scenario, pancreatic nerves react when they are surrounded and invaded by inflammatory cells.

\section{EXTRAPANCREATIC CAUSES OF PAIN} (table 1)

Bile duct stenosis and duodenal stenosis due to extensive pancreatic fibrosis and inflammation have been considered as extrapancreatic causes of pain. ${ }^{12}{ }^{13}$ Becker and Mischke described a pathological condition named "groove pancreatitis", in $19.5 \%$ of 600 patients with $\mathrm{CP}^{14}$ This is characterised by the formation of a scar plate between the head of the pancreas and the duodenum. A scar in the groove is said to lead to complications that are determined by topography: disturbance in the motility of the duodenum, stenosis of the duodenum, and tubular stenosis of the common bile duct, occasionally leading to obstructive jaundice. These alterations are suggested to be responsible for several symptoms present in CP and for postprandial pain due to compression of nerves and ganglia located between the pancreatic head and the duodenum. ${ }^{15}$

\section{PANCREATIC CAUSES OF PAIN (table 1)} Increased intrapancreatic pressure

Intrapancreatic pressure may be related to secretion in the presence of an obstruction to the pancreatic duct. ${ }^{16}$ Altering obstruction and secretion can modulate pain intensity and frequency. ${ }^{17}{ }^{18}$ In fact, many investigators have related the origin of pain to increased pressure in pancreatic ducts and tissue. ${ }^{19}$ The ductal hypertension hypothesis as an explanation for pain in CP is supported by observations that decompression of a dilated pancreatic duct or pseudocyst frequently relieves pain. ${ }^{20}$ Pancreatic enzyme supplementation may also relieve pain in some CP patients. ${ }^{21}$ It is believed

Abbreviations: $\mathrm{CP}$, chronic pancreatitis; $\mathrm{CCK}$ cholecystokinin; CGRP, calcitonin gene related peptide; $\mathrm{SP}$, substance $\mathrm{P} ; \mathrm{NGF}$, nerve growth factor; NK-1R, neurokinin 1 receptor; IL, interleukin; GAP-43, growth associated protein 43 ; TrkA, tyrosine kinase $A$. 
Table 1 Pathogenesis of pain in chronic pancreatitis: different hypotheses

\begin{tabular}{ll}
\hline Pancreatic causes & Extrapancreatic causes \\
\hline $\begin{array}{l}\text { Increased pressure in ducts and } \\
\text { pancreatic tissue }\end{array}$ & Duodenal stenosis \\
Pancreatic ischaemia & Common bile duct stenosis \\
Pancreatic fibrosis & Maldigestion \\
Pancreatic pseudocysts & \\
Acute inflammation of the pancreas & \\
Alteration of pancreatic nerves & \\
Neuroimmune interaction & \\
\hline
\end{tabular}

that the beneficial effects of pancreatic enzymes are related to cholecystokinin (CCK) mediated feedback regulation of pancreatic exocrine secretion by the activity of proteases in the lumen of the small intestine. ${ }^{21}$ According to this hypothesis, administration of pancreatic enzymes reduces hypercholecystokininaemia in patients with $\mathrm{CP}$, resulting in less stimulation of the pancreas, producing lower intraductal pressure, and thereby reducing pain. ${ }^{21}$ Interestingly, pancreatic insufficiency appearing several years after a diagnosis of CP may be accompanied by reduction or complete relief of pain, thus suggesting that the disease can burn itself out. ${ }^{12}$ Amman and colleagues ${ }^{9}$ observed pain relief a median of 4.5 years after onset. Pain relief was accompanied by a marked increase in pancreatic dysfunction and calcifications. However, the perception that the painful pancreas will burn out itself is not supported by other studies. ${ }^{11}$ The burn out theory in CP has been questioned by epidemiological data which show that pain in many patients with CP continues despite pancreatic insufficiency, the appearance of calcifications, alcohol withdrawal, or pancreatic surgery. It has been estimated that approximately $30 \%$ of patients treated with decompressive surgery exhibit recurrent attacks of pain. ${ }^{223}$

\section{"Many investigators have related the origin of pain to increased pressure in pancreatic ducts and tissue"}

Observations are not all consistent with a secretion-pain relationship. Induction of pancreatic secretion by secretin, CCK, or caerulein, as usually done in standard pancreatic function tests (Lundh or serum pancreolauryl test) is not associated with pain in $\mathrm{CP}$ patients. In fact, octreotide, a somatostatin analogue which strongly inhibits pancreatic secretion and therefore should interrupt this postulated pain cycle described above, failed to significantly reduce the pain syndrome in many patients with chronic pancreatitis. ${ }^{24-26}$

Ebbehoj ${ }^{18}$ reported a direct relationship between pain intensity and intraductal pancreatic pressure before and after decompressive surgery. In contrast with this study, Manes and colleagues $^{21}$ found no relationship between pain score and pancreatic pressure although intrapancreatic pressure was positively correlated with ductal changes. Pancreatic pressure was significantly higher in $\mathrm{CP}$ than in controls. Postoperatively, pancreatic pressure decreased by $15.3 \%$ in four patients with CP in whom pressure assessment was repeated after surgical decompression. They concluded that pancreatic parenchymal pressure is not closely related to pain in $\mathrm{CP}^{21}$

\section{Pancreatic ischaemia}

Another hypothesis suggests that pain is induced when increased pancreatic ductal and parenchymal pressure produce a compartment syndrome that causes ischaemia. ${ }^{7}$ This hypothesis is supported by experimental studies ${ }^{72}$ that show that increased interstitial pressure correlates with decreased blood flow in a feline model of chronic pancreatitis. These abnormalities were reversed by surgical incision of the gland and draining the pancreatic duct but were affected minimally by stenting the pancreatic duct. This would suggest that incision of the gland may be more important in relieving pain than ductal drainage.

\section{Pancreatic fibrosis}

$\mathrm{CP}$ is characterised by the presence of intra- and perilobular fibrosis that leads to irreversible scarring. The pathogenesis of pancreatic fibrogenesis is still unclear but a common concept is that fibrosis leads to increased intraductal pressure in the chronically inflamed pancreas and thereby to pain during the course of $\mathrm{CP}^{28}$ However, recent studies ${ }^{29}$ revealed that the degree of pancreatic fibrosis has no significant influence on pain generation as no correlation between the degree of fibrosis and intensity of pain could be demonstrated.

\section{Pancreatic pseudocysts}

Pseudocysts of the pancreas can cause intense pain in CP patients. In the majority of cases $(60 \%)$ treatment with octreotide results in a reduction in size and in eventual disappearance of the pseudocysts together with reduction in pain. ${ }^{30}$ Enlargement of pseudocysts, causing compression of adjacent structures, might be a mechanism for pain generation.

\section{INFLAMMATION IN THE PANCREAS (table 2) \\ Acute inflammation}

Acute inflammation may develop in a chronically diseased pancreas. Whether or not acute pancreatitis may progress to the chronic form is still a subject of controversy. In many patients recurrent attacks of acute inflammation lead to severe abdominal pain. The inflammatory process, involving activated enzymes and other injurious substances, could be responsible for pain generation.

A recent report showed increased expression of the neurotrophin nerve growth factor (NGF) during the course of experimental acute pancreatitis in the rat. ${ }^{31}$ In human $\mathrm{CP}$, neurotrophin gene expression correlates with the intensity of pain. ${ }^{32}$ Comparing these data we can speculate that similar pathogenetic mechanisms operate. However, this possibility should be investigated further.

\section{Alteration of pancreatic nerves}

A current concept of the pathogenesis of pain in CP involves interaction of the nervous system and the inflammatory process as crucial factors. Supporting this hypothesis, Keith et al suggested that neural and perineural alterations may be important in pain pathogenesis in $\mathrm{CP}^{33}$ They concluded that pain severity correlated with duration of alcohol consumption, pancreatic calcification, and with the percentage of eosinophils in perineural inflammatory cell infiltrates, but not with duct dilatation.

"A current concept of the pathogenesis of pain in CP involves interaction of the nervous system and the inflammatory process as crucial factors"

A subsequent study demonstrated an increase in both the number and diameter of pancreatic nerve fibres in the course of $\mathrm{CP}^{34}$ In tissue specimens from patients suffering from $\mathrm{CP}$, foci of chronic inflammatory cells were often found surrounding pancreatic nerves, which by electron microscopic analysis exhibit a damaged perineurium and invasion by lymphocytes. These abnormalities might allow free access of inflammatory mediators or active pancreatic enzymes into nerves, generating and sustaining pain. The changed pattern of intrinsic and possibly extrinsic innervation of the pancreas in CP suggested that there could be upregulation of neuropeptides that usually populate those enlarged nerves. In fact, a further study showed $^{35}$ that there were striking changes in peptidergic nerves in CP. The changes consisted of intensification of 
Table 2 Pathogenesis of pain in chronic pancreatitis: neuroimmune interaction

\begin{tabular}{|c|c|}
\hline Study & Findings \\
\hline Keith et al $1985^{33}$ & $\begin{array}{l}\text { Pain correlated with perineural eosinophils in chronic } \\
\text { pancreatitis }\end{array}$ \\
\hline Bockman et al $1988^{34}$ & $\begin{array}{l}\text { Inflammatory foci; damage to perineurium; more enlarged } \\
\text { nerves }\end{array}$ \\
\hline Büchler et al $1992^{35}$ & Increased neuropeptide expression in chronic pancreatitis \\
\hline Fink et al $1994^{36}$ & $\begin{array}{l}\text { Growth associated protein } 43 \text { (GAP- } 43 \text { ) expression and } \\
\text { neuronal sprouting }\end{array}$ \\
\hline Di Sebastiano et al $1997^{29}$ & $\begin{array}{l}\text { Correlation between GAP- } 43 \text { expression, immune cell } \\
\text { infiltration, and pain }\end{array}$ \\
\hline Friess et al $1999^{32}$ & $\begin{array}{l}\text { Nerve growth factor and its high affinity receptor, the } \\
\text { tyrosine kinase } A(T r k A) \text { receptor, in chronic pancreatitis } \\
\text { correlates with pain intensity }\end{array}$ \\
\hline Di Sebastiano et al $2000^{41}$ & Increased interleukin 8 gene expression \\
\hline Shrikande, et al $2001^{40}$ & Relation between substance $P$ receptor and pain \\
\hline Zhu et al $2001^{37}$ & $\begin{array}{l}\text { Brain derived neurotrophic factor increased expression in } \\
\text { chronic pancreatitis correlates with pain score }\end{array}$ \\
\hline
\end{tabular}

immunostaining for calcitonin gene related peptide (CGRP) and substance P (SP) in numerous nerve fibres. Furthermore, double fluorescence immunohistochemistry revealed the coexistence of SP and CGRP immunoreactive nerves. Because both of these peptides are generally regarded as pain neurotransmitters, these findings provide evidence for direct involvement of pancreatic nerves in the long lasting pain syndrome in $\mathrm{CP}$.

\section{Neuroimmune interaction}

Studies of nerves brought into focus the close spatial relationship between neuronal structures and immune cells in $\mathrm{CP}$, leading to the concept of neuroimmune mechanisms in the pathogenesis of $\mathrm{CP}$ and the accompanying abdominal pain.

\section{Neuronal plasticity and clinical findings}

Subsequent reports ${ }^{29}{ }^{36}$ revealed that the presence of growth associated protein 43 (GAP- 43), an established marker of neuronal plasticity, directly correlated with pain scores in patients with CP. GAP-43 is a neuronal protein known to be involved in the development of axonal growth cones and presynaptic terminals, and mRNA and protein levels of GAP-43 are increased after neuronal lesions. GAP-43 is widespread in both the developing and adult central and peripheral nervous systems of the rat and is expressed in the hippocampus of rats and humans, regions which continually undergo synaptic remodelling after nerve damage. In the chronically inflamed human pancreas, enzymatic and double fluorescence immunohistochemistry reveals significant expression of GAP-43 in the majority of pancreatic nerve fibres.

\begin{abstract}
"Demonstration of a direct relationship between the degree of perineural inflammation and the clinical pain syndrome strongly supports the hypothesis of neuroimmune interaction as an important, if not predominant, factor in pain generation in CP patients"
\end{abstract}

These immunohistochemical findings correlated with clinical and pathological findings in CP patients, including the parenchyma-fibrosis ratio and the degree of perineural immune cell infiltration. Furthermore, a strong relationship with individual pain scores was present. Infiltration of pancreatic nerves by immune cells is significantly related to pain intensity whereas pain scores do not correlate with the degree of pancreatic fibrosis or with duration of disease. Demonstration of a direct relationship between the degree of perineural inflammation and the clinical pain syndrome strongly supports the hypothesis of neuroimmune interaction as an important, if not predominant, factor in pain generation in CP patients.

\section{Nerve growth and pain}

An interesting question concerns the mechanisms that contribute to the enlargement of pancreatic nerves. A recent study analysed expression of NGF and one of its receptors (tyrosine kinase A (TrkA)) in patients suffering from CP. ${ }^{32}$ NGF belongs to the neurotrophin family and plays a role in neuroblast proliferation and neuronal maturation, affecting neuronal phenotype and maintaining neuronal survival. NGF signalling is mediated via binding to high and low affinity receptors. The high affinity receptor is called TrkA, and signalling is transmitted via an internal tyrosine kinase domain. TrkA is present in dorsal root and peripheral ganglia cells of primary sensory nerves, and is involved in signal transduction of noxious stimuli and tissue injury. Inflammation results in an elevation in NGF levels in different diseases.

"The NGF/TrkA pathway is activated in CP and this activation might influence nerve growth and the pain syndrome"

Interestingly, NGF may itself have cytokine-like functions; it can modify mast cell, macrophage, and B cell functions but may also activate TrkA located on sensory and sympathetic nerve fibres innervating the site of inflammation, thus modulating neuroimmune interactions. In CP tissue samples, NGF and TrkA mRNA expression are markedly increased and enhanced. NGF mRNA expression is present in ductal cells, in degenerating acinar cells, and in acinar cells dedifferentiating into tubular complexes. TrkA mRNA is prominent in the perineurium. Enhanced NGF and TrkA mRNA signals are also present in intrapancreatic ganglion cells in CP. Comparison of the molecular findings with clinical parameters revealed a significant relationship between NGF mRNA levels and pancreatic fibrosis and acinar cell damage, and between TrkA mRNA levels and pain intensity. These findings indicate that the NGF/TrkA pathway is activated in CP and that this activation might influence nerve growth and the pain syndrome, most probably by modulating the sensitivity of NGF independent primary sensory neurones through increasing channel and receptor expression. ${ }^{32}$ Similar results, showing a positive correlation with pain intensity and frequency in patients suffering from CP, were reported for brain derived neurotrophic factor gene expression, a member of the neurotrophin family. ${ }^{37}$

\section{Neuroimmune cross talk}

Other mechanisms by which upregulated NGF might influence the pain syndrome in CP patients include regulation of transcription and synthesis of SP and CGRP, as well as by 
release of histamine. The neuropeptide SP is the main tachykinin involved in neural transmission of sensory information, smooth muscle contraction, nociception, sexual behaviour, and possibly wound healing and tissue regeneration. ${ }^{38}{ }^{39}$ SP has wide ranging functional effects, including cross talk between nervous and immune systems by acting through its specific receptor, neurokinin l (NK-lR). A recent report by Shrikande and colleagues ${ }^{40}$ demonstrated a significant correlation between NK-1R and clinical-pathological findings in CP patients. In CP samples, NK-1R mRNA expression and protein were localised mainly in nerves, ganglia, blood vessels, inflammatory cells, and occasionally in fibroblasts. A significant relationship between NK-1R mRNA levels and intensity, frequency, and duration of pain in CP patients, but not with the degree of tissue inflammation, was reported. Expression of NK-1R in inflammatory cells and blood vessels also points to cross talk between immunoreactive SP nerves and inflammatory cells and blood vessels, and further supports the existence of a neuroimmune interaction that probably influences the pain syndrome and chronic inflammatory changes in CP.

\section{Neuropeptides and cytokines}

The exact mechanisms involved in the interaction between inflammatory cells and nerves and ganglia-neuroimmune cross talk-are not yet fully clarified. Different cytokines have been shown to interact with SP in various paradigms for pain and inflammation. Interleukin (IL) 1 and SP increase the proliferation of a fibroblast cell line synergistically. SP directly stimulates the release of IL- 8 from macrophages. IL-8 release generates hyperalgesia by stimulation of post-ganglionic sympathetic neurones. A significant increase in IL-8 mRNA was reported in CP tissue samples. ${ }^{41}$ IL- 8 was present mainly in macrophages surrounding the enlarged pancreatic nerves, in remaining acinar cells, and often in ductal cells. IL-8 mRNA expression was positively correlated with the inflammatory score and the presence of ductal metaplasia in CP tissue samples.

\section{"The exact mechanisms involved in the interaction between inflammatory cells and nerves and ganglia- neuroimmune cross talk - are not yet fully clarified"}

The proinflammatory cytokine $\mathrm{IL}-8$ is a well known $\alpha$-chemokine involved in leucocyte recruitment and activation, and it is representative of a family of factors. The reported findings in the literature on the interaction of SP and IL-8, in combination with what was reported in $\mathrm{CP}$, suggests that increased mRNA expression of IL-8 in CP could in part be mediated by SP released from sensory pancreatic nerves. This speculation is supported by considering that a major source of IL-8 is inflammatory cells present around enlarged nerves in the inflammatory foci. Thus induction of IL-8 in immune cells by SP might contribute to amplification of the inflammatory process in CP. In addition, release of IL-8 from the remaining exocrine pancreatic parenchyma suggests the fascinating hypothesis of intrinsic maintenance of the inflammatory response after the first damage to the pancreatic gland, thus sustaining progression and evolution of the disease.

\section{CONCLUSION}

There are several hypotheses concerning pain pathophysiology in CP. However, the mechanism for the generation and continuation of chronic pain and inflammation in $\mathrm{CP}$ remains somewhat unclear. Involvement of neuropeptides released from enteric and afferent neurones, and their functional interactions with inflammatory cells, may play a key role. The selective increase in the density of tachykinin receptors in the bowel of patients suffering from Crohn's disease and ulcerative colitis provides evidence that SP can be involved in painful chronic inflammatory disorders of the gastrointestinal system. ${ }^{42-45}$ The most pathophysiologically important aspect is the presence of close interrelations between peptidergic neurones and inflammatory cells in CP. Furthermore, there is the intriguing possibility of functional interaction among neuropeptides, immune cells, cytokines, and nerve growth factors. The possible importance of such a constellation in sustaining pain and inflammation has been suggested. ${ }^{46}{ }^{47} \mathrm{~A}$ correlation between immunohistochemical data and pain has been demonstrated in CP. Taken together, the present information provides evidence for neuroimmune cross talk in the pathogenesis of pain and inflammation in CP.

"Further studies are needed to clarify the interaction of inflammatory cells and nerves in CP"

Further studies are needed to clarify the interaction of inflammatory cells and nerves in CP. Simultaneously, efforts should be directed towards developing a relevant experimental model of CP that mimics the human disease. If a clinically relevant experimental $\mathrm{CP}$ model can be established, it will allow further evaluation of the effects of SP receptor antagonists on pain and inflammation in this disorder, in line with similar attempts already performed in other experimental models of inflammatory disorders of the gastrointestinal tract. $^{47-49}$

A better understanding of the complex cellular and molecular mechanisms of neuroimmune interactions should offer possibilities for innovative therapy and long term disease prevention.

\section{Authors' affiliations}

P Di Sebastiano, F F di Mola, D E Bockman, H Friess, M W Büchler, Department of General Surgery, University of Heidelberg, Germany

\section{REFERENCES}

1 Steer ML, Waxman I, Freedman S. Chronic pancreatitis. N Engl J Med 1995:332:1482-90.

2 Büchler MW, Friess $\mathrm{H}, \mathrm{Uhl}$ W, et al. Chronic pancreatitis: Novel Concepts in Biology and Therapy. Oxford: Blackwell Publishing, 2002

3 Beger HG, Büchler M, Malfertheiner P. Standards in Pancreatic Surgery. New York: Springer-Verlag, 1993.

4 Warshaw AL, Banks PA, Fernandez-Del, et al. AGA Technical review: treatment of pain in chronic pancreatitis. Gastroenterology 1998:115:765-76.

5 Beger HG, Krautzberger W, Bittner R, et al. Duodenum-preserving resection of the head of the pancreas in patients with severe chronic pancreatitis. Surgery 1985;97:467-73.

6 Glasbrenner B, Adler G. Evaluating pain and the quality of life in chronic pancreatitis. Int J Pancreatol 1997:22:163-70

7 Reber HA, Karanjia ND, Alvarez C, et al. Pancreatic blood flow in cats with chronic pancreatitis. Gastroenterology 1992;103:652-9.

8 Karaniia ND, Singh SM, Widdison AL, et al. Pancreatic ductal and interstitial pressure in cats with chronic pancreatitis. Dig Dis Sci 1992;37:268-73

9 Amman RW, Muellhaupt B, Zürich Pancreatitis Study Group. The natural history of pain in alcoholic chronic pancreatitis. Gastroenterology 1999:116:1 132-40.

10 Jensen AR, Matzen P, Malchow-Moller A, et al. Pattern of pain, duct morphology and pancreatic function in chronic pancreatitis: a comparative study. Scand J Gastroenterol 1984;19:334-8.

11 Malfertheiner $\mathbf{P}$, Büchler $M$, Stanescu A, et al. Pancreatic morphology and function in relationship to pain in chronic pancreatitis. Int $J$ Pancreatol 1987:2:59-66.

12 Lankisch PG, Lohr-Happe A, Otto J, et al. Natural course of chronic pancreatitis. Pain, exocrine and endocrine pancreatic insufficiency and prognosis of the disease. Digestion 1993;54:148-55.

13 Levy P, Lesur G, Belghiti J, et al. Symptomatic duodenal stenosis in chronic pancreatitis: a study of 17 cases in a medical surgical series of 306 patients. Pancreas 1993:8:563-7.

14 Becker V, Mischke U. Groove pancreatitis. Int J Pancreatol $1991 ; 10: 173-82$

15 Prinz RA, Aranha GV, Greenlee HB, et al. Common duct obstruction in patients with intractable pain of chronic pancreatitis. Am Surg 1982:48:373-7.

16 Manes G, Pieramico O, Uomo G. Pain in chronic pancreatitis: recent pathogenetic findings. Minerva Gastroenterol Dietol 1992;38:137-43.

17 Bradley EL 3rd. Pancreatic duct pressure in chronic pancreatitis. Am J Surg 1982;144:313-16. 
18 Ebbehoj N. Pancreatic tissue fluid pressure and pain in chronic pancreatitis. Dan Med Bull 1992;39:128-33.

19 Ebbehoj N, Borly L, Bulow J, et al. Pancreatic tissue fluid pressure in chronic pancreatitis. Relation to pain, morphology, and function. Scand J Gastroenterol 1990:25:1046-51.

20 Ebbehoi N, Borly L, Madsen P, et al. Pancreatic tissue fluid pressure during drainage operations for chronic pancreatitis. Scand Gastroenterol 1990;25:1041-5.

21 Manes G, Buchler M, Pieramico O, et al. Is increased pancreatic pressure related to pain in chronic pancreatitis? Int J Pancreatol 1994:15:113-17.

22 Beger HG, Schlosser W, Friess HM, et al. Duodenum-preserving head resection in chronic pancreatitis changes the natural course of the disease: a single-center 26-year experience. Ann Surg 1999;230:512-19

23 Ihse I, Borch K, Larsson J. Chronic pancreatitis: results of operations for relief of pain. World J Surg 1990;14:53-8

24 Uhl W, Anghelacopoulos SE, Friess $\mathrm{H}$, et al. The role of octreotide and somatostatin in acute and chronic pancreatitis. Digestion 1999;60/suppl 2):23-31

25 Malfertheiner $\mathbf{P}$, Mayer $\mathrm{D}$, Buchler $\mathrm{M}$, et al. Treatment of pain in chronic pancreatitis by inhibition of pancreatic secretion with octreotide. Gut. 1995;36:450-4.

26 Di Magno EP. Toward understanding (and management) of painful chronic pancreatitis. Gastroenterology 1999;116:1252-7.

27 Karaniia ND Widdison AL, Leung F, et al Compartment syndrome in experimental chronic obstructive pancreatitis: effect of decompressing the main pancreatic dust. BrJ Surg 1994;81:259-64.

28 di Mola FF, Friess H, Martignoni ME, et al. Connective tissue growth factor is a regulator for fibrosis in human chronic pancreatitis. Ann Surg 1999;230:63-71.

29 Di Sebastiano $\mathbf{P}$, Fink T, Weihe $\mathrm{E}$, et al. Immune cell infiltration and growth-associated protein 43 expression correlate with pain in chronic pancreatitis. Gastroenterology 1997; 112:1648-55

30 Gullo L, Barbara L. Treatment of pancreatic pseudocysts with octreotide. Lancet 1991;338:540-41.

31 Toma H, Winston J, Micci MA, et al. Nerve growth factor expression is up-regulated in the rat model of L-arginine-induced acute pancreatitis. Gastroenterology 2000;11 19:1373-81.

32 Friess H, Zhu ZW, di Mola FF, et al. Nerve growth factor and its high affinity receptor in chronic pancreatitis. Ann Surg 1999.230:615-24.

33 Keith RG, Keshavjee SH, Kerenyi NR. Neuropathology of chronic pancreatitis in humans. Can J Surg 1985;28:207-11.

34 Bockman DE, Buchler $M$, Malfertheiner $P$, et al. Analysis of nerves in chronic pancreatitis. Gastroenterology 1988;94:1459-69.
35 Buchler $M$, Weihe $E$, Friess $H$, et al. Changes in peptidergic innervation in chronic pancreatitis. Pancreas 1992:7:183-92.

36 Fink T, Di Sebastiano P, Büchler M, et al. Growth associated protein-43 and protein gene product 9.5 innervation in human pancreas: changes in chronic pancreatitis. Neuroscience 1994;63:249-66.

37 Zhu ZW, Friess H, Wang L, et al. Brain-derived neurotrophic factor (BDNF) is upregulated and associated with pain in chronic pancreatitis. Dig Dis Sci 2001;46:1633-9.

38 Di Sebastiano P, Weihe E, di Mola FF, et al. Neuroimmune appendicitis. Lancet 1999;354:461-6.

39 Weihe E, Nohr D, Muller S, et al. The tachykinin neuroimmune connection in inflammatory pain. Ann N Y Acad Sci 1991:632:283-95.

40 Shrikande S, Friess H, di Mola FF, et al. NK-1 receptor gene expression is related to pain in chronic pancreatitis. Pain 2001;91:209-17.

41 Di Sebastiano P, di Mola FF, Di Febbo C, et al. Expression of interleukin-8 (IL-8) and substance P in human chronic pancreatitis. Gut 2000;47:423-8.

42 Mantyh CR, Gates TS, Zimmerman RP, et al. Receptor binding sites for substance $P$, but not substance $K$ or neuromedin $K$, are expressed in high concentration by arterioles, venules, and lymph nodules in surgical specimen obtained from patients with ulcerative colitis and Crohn's disease. Proc Natl Acad Sci U S A 1988;85:3235-9.

43 Mantyh PW, Catton MD, Boehmer CG et al. Receptor for sensory neuropeptides in human inflammatory diseases: implications for the effector role of sensory neurons. Peptides 1989;10:627-45.

44 Mantyh PW, Mantyh CR, Gates T, et al. Receptor binding sites for substance $\mathrm{P}$ and substance $\mathrm{K}$ in the canine gastrointestinal tract and their possible role in inflammatory bowel disease. Neuroscience 1988:25:817-37.

45 Di Sebastiano P, Grossi L, di Mola FF, et al. SR 140333, a substance P receptor antagonist, influences morphological and motor changes in rat experimental colitis. Dig Dis Sci 1999;44:439-44.

46 Stanisz AM, Stanisz JA. Nerve growth factor and neuroimmune interactions in inflammatory diseases. Ann N Y Acad Sci 2000;917:268-72

47 Levi-Montalcini R, Skaper SD, Dal Toso R, et al. Nerve growth factor from neurotrophin to neurokine. Trends Neurosci 1996;19:514-20.

48 Jafarian-Tehrani $M$, Sternberg EM. Animal models of neuroimmune interactions in inflammatory diseases. J Neuroimmunol $1999 \cdot 100 \cdot 13-20$.

49 Di Sebastiano P, di Mola FF, Artese L, et al. Beneficial effects of BB-94, a matrix metalloproteinase inhibitor, in rat experimental colitis. Digestion $2001 ; 63: 234-9$. 